\title{
BAIXA VACINAÇÃO PARA BRUCELOSE EM BEZERRAS BOVINAS (Bos taurus) E BUBALINAS (Bubalos bubalis) EM 7 MUNICÍPIOS DO ESTADO DE ALAGOAS, BRASIL NO PERÍODO DE JUNHO DE 2015 A JUNHO DE 2018
}

\author{
Abelardo Mesquita Remigio Neto ${ }^{1}$ \\ José Vicente Ferreira Neto ${ }^{2}$ \\ Rodrigo Antônio Torres Matos ${ }^{3}$
}

\begin{abstract}
RESUMO
O presente estudo tem como objetivo avaliar os índices inseridos no banco de dados da Agência de Defesa e Inspeção Agropecuária do Estado de Alagoas, Unidade Local de Sanidade Animal e Vegetal de Maceió, sobre cobertura vacinal da Brucelose em bezerras de três à oito meses de idade, no período de junho de 2015 à junho de 2018. Para comparação entre bezerras vacináveis e vacinadas, foi utilizado o teste $\mathrm{T}$ de student $\mathrm{e}$ as análises foram realizadas com o programa estatístico Graph Pad. Tendo em vista o total de bezerras em idade de vacinação, observou-se que 26,01\% (5.021/19.299) delas foram vacinadas contra brucelose bovina, durante o período do estudo, de acordo com os registros de vacinação dos criadores. Em relação ao número de bezerras vacináveis e bezerras vacinadas, houve diferença estatisticamente significante na comparação entre esses dois grupos. Visto este cenário, a conscientização do pecuarista é de extrema importância, uma vez que o mesmo é peça fundamental nas ações coordenadas pelos órgãos de defesa sanitária e a sua participação irá contribuir de forma expressiva para a elevação dos índices de imunização do rebanho.
\end{abstract}

Palavras-chave: Defesa sanitária, índices, pecuarista, imunização.

LOW VACCINATION BRUCELLOSIS IN BOVINE HEIFERS (Bos taurus AND Bubalos bubalis) IN 7 MUNICIPALITIES IN THE STATE OF ALAGOAS, BRAZIL IN THE PERIOD FROM JUNE 2015 TO JUNE 2018

\begin{abstract}
The present study aims to evaluate the indexes inserted in the Agência de Defesa e Inspeção Agropecuária do Estado de Alagoas database, Unidade Local de Sanidade Animal e Vegetal de Maceió, on vaccination coverage of Brucellosis in calves from three to eight months of age, from June 2015 to June 2018. For comparison between vaccinate and vaccinated calves, the Student $\mathrm{T}$ test was used and the analyzes were performed with the Graph Pad statistical program. In view of the total number of calves in the vaccination age, it was observed that $26.01 \%(5,021 / 19,299)$ of them were vaccinated against bovine brucellosis, during the study period, according to the vaccination records of the breeders. Regarding the number of vaccinable calves and vaccinated calves, there was a statistically significant difference in the comparison between these two groups. In view of this scenario, raising ranchers' awareness is extremely important, since it is a fundamental part of the actions coordinated by health defense agencies and their participation will contribute significantly to the increase in the herd's immunization rates.
\end{abstract}

Keywords: Health defense, index, rancher, immunization.

\footnotetext{
1 Agência de Defesa e Inspeção Agropecuária do Estado de Alagoas Maceió, Alagoas, Brasil vicentemedvet@gmail.com

2 Discente do Programa de Pós-Graduação em Ciências Biológicas (Entomologia), Instituto Nacional de Pesquisas da Amazônia, Manaus, Amazonas. Laboratório Ecologia de Doenças Transmissíveis na Amazônia, Instituto Leônidas e Maria Deane - Fiocruz Amazônia, Manaus, Amazonas, Brasil. Correspondência: vicentemedvet@gmail.com 3 Centro Universitário CESMAC, Maceió, Alagoas, Brasil. rodrigomatos@gmail.com
}

Remigio Neto AM, Ferreira Neto JV, Matos RAT. Baixa vacinação para brucelose em bezerras bovinas (Bos taurus) e bubalinas (Bubalos bubalis) em 7 municípios do estado de Alagoas, Brasil no período de junho de 2015 a junho de 2018. Vet. e Zootec. 2021.; v28: 001-006. 


\section{BAJA VACUNACIÓN CONTRA BRUCELOSIS EN TERNEIRAS (Bos taurus Y Bubalos bubalis) EN 7 MUNICIPIOS DEL ESTADO DE ALAGOAS, BRASIL EN EL PERIODO DE JUNIO DE 2015 A JUNIO DE 2018}

\section{RESUMEN}

El presente estudio tiene como objetivo evaluar los índices ingresados en la base de datos Agência de Defesa e Inspeção Agropecuária do Estado de Alagoas, Unidade Local de Sanidade Animal e Vegetal de Maceió, sobre la cobertura de vacunación contra la brucelosis en terneros de tres a ocho meses de edad, en el período de junio de 2015 a junio de 2018. Para la comparación entre terneros vacunados y vacunados, se utilizó la prueba t de Student y los análisis se realizaron con el programa estadístico Graph Pad. Considerando el total de terneros en edad de vacunación, se observó que el 26,01\% (5.021 / 19.299) de ellos fueron vacunados contra la brucelosis bovina durante el período de estudio, según los registros de vacunación de los criadores. En cuanto al número de vaquillas vacunadas y vacunadas, hubo una diferencia estadísticamente significativa al comparar estos dos grupos. Ante este escenario, la sensibilización del ganadero es de suma importancia, ya que es un actor clave en las acciones coordinadas por los organismos de defensa de la salud y su participación contribuirá significativamente a elevar las tasas de inmunización del rebaño.

Palabras: Defensa de la salud, índices, ganadero, inmunización.

\section{INTRODUÇÃO}

A brucelose é uma zoonose de distribuição cosmopolita, causada por bactérias do gênero Brucella ssp. Afeta várias espécies de animais, que podem apresentar sinais clínicos como aborto, retenção de placenta, orquite e infertilidade. O ser humano (Homo sapiens) pode se contaminar através da ingestão ou contato com secreções de animais infectados (1).

A primeira campanha nacional de combate à doença implementada no Brasil, ocorreu em 1975, demostrando uma prevalência de 4,1\%, com 4\% de animais soropositivos na Região Sul, 6,8\% no Centro-Oeste, 7,5\% no Sudeste, 4,1\% no Norte e 2,5\% no Nordeste. E em 2011 foram estimados prejuízos econômicos de aproximadamente $\mathrm{R} \$ 892$ milhões (2).

Com a premissa de controlar e erradicar a Brucelose no Brasil, o Ministério da Agricultura, Pecuária e Abastecimento (MAPA) instituiu, em 2000, o Programa Nacional de Controle e Erradicação da Brucelose e Tuberculose (PNCEBT), que tem o intuito de direcionar e padronizar as ações do serviço oficial de defesa sanitária animal (3).

O Estado de Alagoas, localizado na região nordeste do Brasil, possui 1,2 milhão de bovinos, com aproximadamente 45 mil propriedades rurais, distribuídos em 102 municípios (4). Com a finalidade de planejar, coordenar, executar e fiscalizar programas de produção, de saúde e de defesa sanitária animal e vegetal, foi criado a Agência de Defesa e Inspeção Agropecuária do Estado de Alagoas (ADEAL). A ADEAL possui 15 Unidades Locais de Sanidade Animal e Vegetal (ULSAV) distribuídas no estado, e cada uma delas possui jurisdição das delegacias em uma determinada distribuição geográfica (5).

Visto a necessidade da imunização dos animais contra a doença, afim de estabelecer medidas estratégicas que visem à melhoria e ampliação ao programa de controle da enfermidade de acordo com as características socioeconômicas e biogeográficas de cada região do país. O presente estudo tem como objetivo avaliar a cobertura vacinal da Brucelose em bezerras bovinas (Bos taurus) e bubalinas (Bubalos bubalis), no período de junho de 2015 à junho de 2018, através dos dados disponibilizados pela ULSAV - Maceió, responsável pelos 
municípios de Maceió, Rio Largo, Messias, Atalaia, Satuba, Santa Luzia do Norte e Coqueiro Seco, no estado de Alagoas, Brasil.

\section{MATERIAL E MÉTODOS}

Foi realizado um levantamento dos dados condensados estaduais dos informes mensais de vacinação contra brucelose e relatório semestral da comprovação de vacinação das bezerras de três à oito meses de idade, no período de junho de 2015 à junho de 2018, inseridos no banco de dados da ADEAL, ULSAV - Maceió, responsável pelos municípios de Maceió, Rio Largo, Messias, Atalaia, Satuba, Santa Luzia do Norte e Coqueiro Seco.

Os dados foram tabulados utilizando o programa Excel para geração de tabela da frequência de fêmeas existentes, vacináveis e vacinadas. Para comparação entre bezerras vacináveis e vacinadas, foi utilizado o teste $\mathrm{T}$ de student, adotando um nível de significância de $5 \%$ e as análises foram realizadas com o programa estatístico Graph Pad.

\section{RESULTADOS E DISCUSSÃO}

Tendo em vista o total de bezerras em idade de vacinação, observou-se que $26,01 \%$ (5.021/19.299) delas foram vacinadas contra brucelose bovina, durante o período do estudo, de acordo com os registros de vacinação dos criadores. Em relação ao número de bezerras vacináveis e bezerras vacinadas, houve diferença estatisticamente significante na comparação entre esses dois grupos ( $p=0,0002)$, como demonstrados na Tab. 1.

Tabela 1. Cobertura vacinal de bezerras bovinas e bubalinas entre três e oito meses de idade de acordo com o ano, no período de junho 2015 à junho de 2018, nos municípios de Maceió, Rio Largo, Messias, Atalaia, Satuba, Santa Luzia do Norte e Coqueiro Seco, estado de Alagoas, Brasil

\begin{tabular}{cccccc}
\hline & Campanhas & Animais & \multicolumn{2}{c}{ Bezerras } & Vacinados \\
\hline Ano & Semestre & Existente & Vacináveis & Vacinadas & $\%$ \\
\hline 2015 & $2^{\circ}$ & 34171 & 2878 & 664 & $23,07 \%$ \\
2016 & $1^{\circ}$ & 36675 & 3182 & 540 & $16,97 \%$ \\
2016 & $2^{\circ}$ & 39195 & 3283 & 233 & $7,10 \%$ \\
2017 & $1^{\circ}$ & 41076 & 3484 & 592 & $16,99 \%$ \\
2017 & $2^{\circ}$ & 42653 & 3564 & 1665 & $46,72 \%$ \\
2018 & $1^{\circ}$ & 44322 & 2908 & 1327 & $45,63 \%$ \\
\hline Total & \multicolumn{7}{c}{238.092} & 19.299 & 5.021 & $26,01 \%$ \\
\hline
\end{tabular}

Fonte: ADEAL / ULSAV- Maceió (2018).

Observou-se uma variação dos índices de vacinação e verificou-se uma porcentagem abaixo de $25 \%$ de animais vacinados nos referidos anos, com destaque para o ano de 2016 , que obteve aproximadamente $12 \%$ de cobertura vacinal, sendo considerado muito baixo. A variação destes índices pode ser justificada pela ausência de chuvas no estado de Alagoas e demais regiões circunvizinhas, fato este, que levaram alguns municípios a decretarem estado de emergência, tornando-se inviável a vacinação dos animais, pois muitos estavam debilitados e alguns chegaram a óbito.

Resultados semelhantes foram observados no estado Paraíba por Andrade (6), que verificou uma redução no índice de vacinação de bezerras de três a oito meses contra a brucelose, ocorrendo uma diminuição de 7\% em relação ao ano anterior de 2011 devido a um período de escassez de chuvas no estado, fato que corrobora com a observação feita no 
segundo semestre de 2016 deste estudo, revelando que os fenômenos naturais influenciaram negativamente nos índices de vacinação.

Outro fato que merece destaque, ocorrido neste mesmo período de transição entre os anos de 2015 e 2016 foi uma paralisação coletiva das atividades no estado de Alagoas, realizada pelos servidores do órgão. Este episódio inviabilizou o serviço veterinário oficial, prejudicando serviços como: vigilância ativa dos rebanhos, educação sanitária, acompanhamento de campanhas de vacinação, cadastro de produtores e propriedades rurais e controle de trânsito de animais.

Nos anos de 2017 e 2018 os índices passaram a aumentar nos municípios observados. Em 2017, o índice atingiu 32.02\% (2257/7048), uma boa cobertura, quando comparado ao ano anterior em relação a 2018. No primeiro semestre, como é observado na tabela, a cobertura vacinal apresenta 45\% (1327/2908), havendo uma mínima oscilação em relação a 2017. Isso indica que, possivelmente, em 2018, a cobertura vacinal deva ter um resultado satisfatório, já que esta porcentagem atribuída a este ano refere-se apenas ao primeiro semestre. Inlamea et al. (7) cita que a doença está associada à exploração de gado de corte, ao maior número de vacas e a presença de áreas alagadas, mas destaca que a implementação do programa de vacinação, reduz significativamente a prevalência da doença em rebanhos infectados

Observando o aumento da cobertura vacinal neste período de 2017 a 2018, o aumento possivelmente se deve a Instrução Normativa $\mathrm{N}^{\circ} 10$, de março de 2017 . Neste período a ADEAL começou a colocar em prática as exigências desta Instrução Normativa como o aumento da fiscalização em relação à obrigatoriedade da comprovação vacinal. Esta comprovação é realizada por meio da declaração das bezerras bovinas e bubalinas de três a oito meses contra a brucelose. O MAPA institui comprovação a cada semestre, ou seja, dois semestres por ano, esta declaração deve ser realizada pelo criador junto ao serviço veterinário oficial, após a vacinação dos animais.

Outra exigência que passou a ser cobrada neste período e, possivelmente, ajudou a aumentar o índice de vacinação, foi a obrigatoriedade no ato do recebimento pela indústria do leite in natura, da comprovação da vacinação dos animais das propriedades rurais de origem do leite (3). Segundo Deschk et al. (8), em estudos realizados no período de 2010 a 2017, no município de Guaraniaçu, no Estado do Paraná, esta mesma medida foi implementada em 2013 por meio da Portaria $\mathrm{N}^{\circ} 342$, de outubro de 2013, obtendo no ano de 2014 um aumento de 14,4\% nos índices de animais vacinados neste período.

No entanto Andrade (6) afirma que na Paraíba esta medida também surtiu efeitos positivos, porém foi identificado que um número menor de criadores comprovou a vacinação de um número maior de animais, o mesmo explica que esta constatação se deu pelo fato que só os criadores que tinham interesse em fornecer a matéria prima à indústria ou os criadores que precisavam transitar com os animais fizeram a comprovação da vacinação.

Outro fato que merece destaque no Paraná, no período estudado segundo Deschk et al. (8), influenciou de forma positiva no índice, foi a criação da Agência de Defesa Agropecuária do Paraná (ADAPAR) em 2011, que já no ano seguinte de 2012 teve um índice de 73,2\%, próximo ao recomendado pelo MAPA.

Houve também a restrição de trânsito de animais neste período de 2017 a 2018, quando a ADEAL, seguindo a Instrução Normativa, restringiu a emissão da Guia de Trânsito Animal (GTA) para os criadores que possuíam bezerras na idade de vacinação em seu rebanho e que não possuíam a comprovação da vacinação, ficando assim estes criadores proibidos de transitar com os animais do seu rebanho, como também participar de eventos onde tenham aglomerações de animais como feira de gado, leilões, vaquejadas e outros afins.

No estado da Paraíba, segundo Andrade (6), em 2008 foi observada a menor cobertura vacinal no período entre 2008 e 2013, passando a ter melhores resultados nos anos seguintes 
devido a publicação da portaria reforçando a obrigatoriedade da vacinação e também a exigência da comprovação da vacinação no estado por parte do criador, caso o criador não comprove o mesmo fica impedido de transitar com os animais. Situação que fez o índice de vacinação saltar de $13.97 \%$, em 2008, para 20,95\% em 2009.

Mesmo o estado, seguindo estratégias para aumentar o número de animais imunizados contra a doença na área estudada, é fato que os resultados estão muito longe do percentual mínimo exigido pelo PNCEBT de $80 \%$ de animais vacinados. Segundo Amaku et al. (9), com índices baixos, a prevalência leva muito tempo para ser reduzida quando comparado a índices altos, os autores afirmam ainda que para reduzir a 50\% da prevalência, mesmo com índices a $90 \%$, seria necessário uma década para isso ocorrer. Souza et al. (10) retifica que o criador deve fazer o uso de uma vacina que não induza a produção de anticorpos em animais acima de 8 meses de idade, ainda não vacinados com a B19, reduzindo assim a prevalência da doença, pois se aplicada fora da faixa etária preconizada, pode gerar um resultado falsopositivo e os testes sorológicos de referência não distinguem animais vacinados de animais infectados no campo.

\section{CONCLUSÕES}

Apesar dos índices de cobertura vacinal terem aumentado nos últimos anos nos municípios estudados, estes ainda continuam abaixo do mínimo exigido pelo PNCEBT, que é de $80 \%$, fazendo-se necessária a adoção de medidas de consientização e fiscalização sanitária, para que os índices de cobertura vacinal continuem aumentando.

Faz-se de extrema importância a conscientização do pecuarista, uma vez que o mesmo é peça fundamental nas ações coordenadas pelos órgãos de defesa sanitária e a sua participação irá contribuir de forma expressiva para a imunização do rebanho.

\section{REFERÊNCIAS}

1. Mathias LA, Costa M. Brucelose bovina e equina. In: Riet-Correa F, Schild AL, Lemos RAA, Borges JRJ. Doenças de ruminantes e equídeos. 3a ed. São Paulo: Fernovi; 2007. p. 225-40.

2. Santos RL, Martins TM, Borges ÁM, Paixão TA. Economic losses due to bovine brucellosis in Brazil. Pesqui Vet Bras. 2013;33(6):759-64. doi: https://doi.org/10.1590/S0100-736X2013000600012.

3. Brasil. Ministério da Agricultura, Pecuária e Abastecimento - MAPA. Instrução Normativa $\mathrm{n}^{\mathrm{o}}$ 10, de 3 de Março de 2017. Regulamento Técnico do Programa Nacional de Controle e Erradicação da Brucelose e da Tuberculose Animal. Diário Oficial da União. 20 Jun 2017.

4. Associação Brasileira das Indústrias Exportadoras de Carnes [Internet]. Maceió: ABIEC; 2019 [citado 27 Jun 2021]. Disponível em: https://www.jornaldealagoas.com.br/agro/2019/01/26/1014-alagoas-tem-o-20-rebanho-dopais

5. Alagoas. Lei $\mathrm{n}^{0}$ 6.673, de 4 de Janeiro de 2006. Dispõe sobre a criação da agência de defesa e inspeção agropecuária de alagoas - ADEAL, e dá outras providências. Diário Oficial da União. 5 Jan 2006. 
6. Andrade-Silva SM. Estudo da cobertura vacinal de Brucelose bovina em bezerras no estado da Paraíba [trabalho de conclusão de curso]. Areia: Ciências Veterinárias, Universidade Federal da Paraíba; 2018.

7. Inlamea OF, Rocha AB, Ferreira F, Grisi-Filho JHH, Heinemann MB, Dias RA, et al. Effect of vaccination in lowering bovine brucellosis in the state of Rondônia, Brazil. Semina Cienc Agrar. 2016;37(5):3493-504. doi: 10.5433/16790359.2016v37n5Sup12p3493.

8. Deschk DP, Weber LD, Gai VF. Influência das obrigações legais no índice de vacinação da Brucelose em bezerras de 3 a 8 meses no município de Guaraniaçu no decorrer dos anos de 2010 a 2017. Arq Bras Med Vet. 2018;1(1):58-65.

9. Amaku M, Dias RA, Ferreira Neto JS, Ferreira F. Modelagem matemática do controle de Brucelose bovina por vacinação. Arq Bras Med Vet Zootec. 2009;61(1):135-41. doi: https://doi.org/10.1590/S0102-09352009000700017.

10. Souza VAF, Ferreira Neto JS, Amaku M, Dias RA, Telles EO, Grisi-Filho JHH, et al. Mathematical modeling of bovine brucellosis control using the RB51vaccine. Semina Cienc Agrar. 2016;37(5):3767-76. doi: 10.5433/1679-0359.2016v37n5Supl2p3767.

Recebido em: 28/06/2021 Aceito em: 31/08/2021 